\title{
A Case Report, Suspected Megakaryoblastic Transformation In A Chronic Myeloid Leukemia Patient
}

\author{
Dita Mernita Setyawatia ${ }^{\mathrm{a}}$ Paulus Budiono Notopuro ${ }^{\mathrm{b}}$ \\ a dimers88@icloud.com \\ ${ }^{a}$ Clinical Pathology Specialization Programme, Department of Clinical Pathology Faculty of Medicine, Airlangga University-Dr. \\ Soetomo Hospital, Mayjen Prof. Dr. Moestopo street no. 6-8, Surabaya 60286, Indonesia \\ ${ }^{b}$ Department of Clinical Pathology, Faculty of Medicine, Airlangga University-Dr. Soetomo Hospital, Mayjen Prof. Dr. Moestopo street \\ no. 6-8, Surabaya 60286, Indonesia
}

\begin{abstract}
Backgroud: Chronic Myeloid Leukaemia (CML) is a clonal hematopoiesis stem cell disorder, characterised by reciprocal translocation between chromosomes 9 and chromosomes 22, originally named the 'Philadelphia chromosome' $(\mathrm{Ph})$. The megakaryoblastic transformation of a CML is extremely rare, consisting $<3 \%$ of transformed cases.

Case Description: A 68-year-old male presented with a CML history since 2000 and received Imatinib therapy. The patient currently showed anemia and splenomegaly. Laboratory results showed hemoglobin $8.1 \mathrm{~g} / \mathrm{dL}$, leukocyte count $31,740 / \mu \mathrm{L}$, platelet count $7,000 / \mu \mathrm{L}$, Immature Platelet Fraction (IPF) $29.2 \%$, BCR-ABL gene fusion is positive. Bone marrow examination showed CML blastic crisis phase with $45 \%$ blasts which had megakaryoblast morphology so it was concluded as a CML that underwent a transformation into megakaryoblastic. The results of bone marrow aspirate using Sysmex-XN1000 showed a very high IPF value which indicated an increased megakaryopoietic proliferative activity. Immunophenotyping examination showed blast population with CD45 dim, low side scatter, with the expression of CD34+, CD33+, CD13+ corresponding to the megakaryoblast area. Examination of the CD41 and CD61 specific megakaryoblast markers was not performed due to reagent limitations.

Conclusion: A CML patient has been reported with suspected megakaryoblastic transformation based on the bone marrow morphology, immunophenotyping, molecular and elevated IPF examination.
\end{abstract}

Keywords: Chronic myeloid leukemia; megakaryoblastic transformation; BCR-ABL; IPF

\section{Introduction}

Chronic Myeloid Leukemia (CML) is a clonal stem cell hematopoiesis disorder characterized by the presence of the Philadelphia chromosome $(\mathrm{Ph})$, a translocation of chromosome 9 with chromosome 22. The CML has three phases, the chronic phase, the accelerated phase, and the blastic crisis phase. Patients are generally diagnosed in the chronic phase. The vast majority of patients who do not receive therapy progress to the blastic phase, either through the accelerated phase or not (1). Blast crisis phase CML can be diagnosed when there is a blast of $\geq 20 \%$ of the peripheral leukocyte count or nucleated cells in the bone marrow, or when there is a extramedullary blast proliferation. Approximately $70 \%$ of blast lineage cases are myeloid 
(neutrophilic, eosinophilic, basophilic, monocytic, megakaryocytic or erythroid blast) and in 20-30\% lymphoid. The BCR-ABL gene is frequently found in high-risk acute lymphoblastic leukemia (ALL) (2). Fusion of this gene is also carried by $0.5-3 \%$ of all cases of acute myelogenous leukemia (AML). The transformation of blastic crisis phase CML to acute megakaryoblastic leukemia (AMegL) is very rare, consisting $<3 \%$ of transformed cases (1). In this case report, we will report a case with suspected megakaryoblastic transformation in a chronic myeloid leukemia patient.

\section{Case Presentation}

A 68-year-old man came to Dr. Soetomo Surabaya on June 28, 2019 with complaints of weakness, full stomach and reduced appetit. No bleeding gums and nosebleeds. The patient was diagnosed with CML since 2000 with a positive BCR-ABL p210 gene fusion. From 2000 to 2018 patients were given Imatinib therapy then in 2018 to 2019 the therapy was replaced with Tasigna (Nilotinib).

Physical examination on 28 June 2019 found the patient's general condition was weak, GCS 4-5-6, blood pressure $110 / 80 \mathrm{mmHg}$, pulse $83 \mathrm{x} /$ minute, breathing $18 \mathrm{x} /$ minute, axillary temperature $36.8^{\circ} \mathrm{C}$. Examination of the head was anemic, without jaundice, without cyanosis, and without dyspnea. Thoracic examination revealed a symmetrical chest shape, without chest wall retraction. Single S1 S2 heart sound, without gallops or heart murmurs. Breath sounds are vesicular, without crackles and wheezing. Abdominal examination revealed normal bowel sounds, no liver was palpable, there was an enlarged Schuffner's spleen 6, there was no kidney enlargement. Examination of the extremities showed no petechiae, warm, dry extremity without edema.

Complete blood count with Sysmex XN-1000 revealed anemia, leukocytosis, basophilia, thrombocytopenia, high Immature Platelet Fraction (IPF) values plus flagging blast, immature granulocytes present (Table 1). Peripheral blood smear evaluation dated August 6, 2019 (Figure 2), found 76\% micromegakaryocytes, $1 \%$ segment neutrophils, $18 \%$ mature lymphocytes, $3 \%$ monocytes and $2 \%$ basophils suspicious of AML-M7. Bone Marrow Aspiration (BMA) analysis revealed many mononuclear cells that resembled abnormal megakaryoblasts with a proportion of $45 \%$ (Table 2 and Figure 3 ). Immunophenotyping examination revealed a blast population with CD45 dim, low side scatter, with expression of CD34+, CD33+, CD13+ corresponding to the megakaryoblast area (Figure 4). Examination of specific megakaryoblast markers CD41 and CD61 was not performed due to limited reagents.

Table 1. Results of the Hematology Laboratory of Dr. Soetomo Hospital, Surabaya

\begin{tabular}{lccl}
\hline Parameters & $\mathbf{2 8 / 0 6 / 1 9}$ & $\mathbf{0 6 / 0 8} / \mathbf{1 9}$ & Normal Value \\
\hline $\mathrm{Hb}(\mathrm{g} / \mathrm{dL})$ & 8,1 & 8,3 & $13,3-16,6$ \\
\hline $\mathrm{RBC}\left(10^{6} / \mu \mathrm{l}\right)$ & 2,74 & 3,03 & $3,69-5,46$ \\
\hline $\mathrm{Hct}(\%)$ & 24,3 & 24,4 & $41,3-52,1$ \\
\hline $\mathrm{MCV}(\mathrm{fl})$ & 88,7 & 80,5 & $86,7-102,3$ \\
\hline $\mathrm{MCH}(\mathrm{pg})$ & 29,6 & 27,4 & $27,1-32,4$ \\
\hline $\mathrm{MCHC}(\mathrm{g} / \mathrm{L})$ & 33,3 & 34 & $29,7-33,1$ \\
\hline $\mathrm{RDW}(\%)$ & 20,7 & 21,3 & $12,2-14,8$ \\
\hline $\mathrm{WBC}\left(10^{3} / \mu \mathrm{l}\right)$ & 33,44 & 31,4 & $3,37-10$ \\
\hline$\% \mathrm{Eo}$ & 0,2 & 0,1 & $0,6-5,4$ \\
\hline$\% \mathrm{Ba}$ & 3 & 0,7 & $0,3-1,4$ \\
\hline$\% \mathrm{Neu}$ & 11,3 & 8,5 & $39,8-70,5$ \\
\hline$\% \mathrm{Ly}$ & 20,4 & 17,7 & $23,1-49,9$ \\
\hline$\% \mathrm{Mo}$ & 65,1 & 73 & $4,3-10$ \\
\hline $\mathrm{Plt}\left(10^{3} / \mu \mathrm{l}\right)$ & 111 & 7 & $150-450$ \\
\hline$\% \mathrm{IPF}$ & 29,2 & & $1,1-6,1$ \\
\hline
\end{tabular}


Table 2. BMA Examination Results Clinical Pathology Hematology Division

\begin{tabular}{ll}
\hline Cellularity & Hypercellular \\
\hline ME Ratio & $12: 1$ \\
\hline Erythropoiesis system & Decreased activity, proportion 4\% \\
\hline Granulopoiesis system & Increased activity by 51\% proportion \\
\hline $\begin{array}{l}\text { Megakaryopoiesis } \\
\text { system }\end{array}$ & $\begin{array}{l}\text { Increased activity, megakaryocytes are easy to find dysplasia (+), many mononuclear } \\
\text { cells are found that resemble abnormal megakaryoblasts with a proportion of 45\% }\end{array}$ \\
\hline Other cells & No non-hematopoietic cells were found \\
\hline Conclusions & $\begin{array}{l}\text { The bone marrow appearance of patients with a history of CML at this time is } \\
\text { consistent with the picture of a blastic crisis leading to AML-M7. }\end{array}$ \\
\hline
\end{tabular}
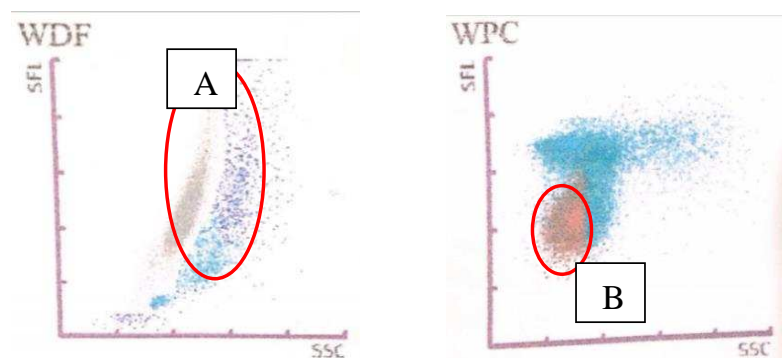

Figure 1. Analysis of White Blood Cell Differential (WDF) channel (right) and White Precursor Cell (WPC) channel (left) on Sysmex $\mathrm{XN}-1000$. (A) The proportion of cells having high fluorescence and moderate complexity suggest a myeloid blast series and immature granulocytes; (B) Proportion of cells showing fluorescence and low complexity, suggesting a myeloid series blast (4).

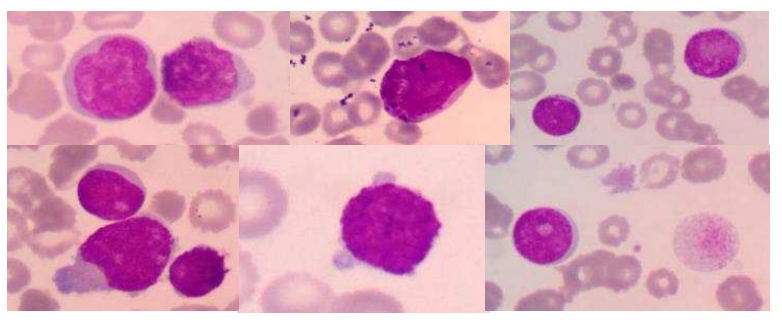

Figure 2. Abnormal micromegakaryocyte morphology in peripheral blood smear patients

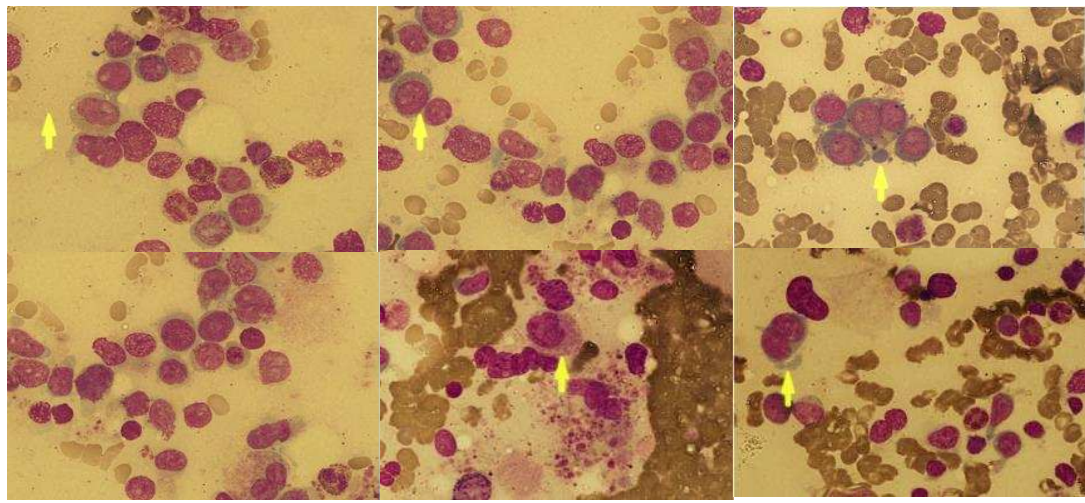

Figure 3. Bone Marrow Aspiration picture shows increased activity of the granulopoiesis system, thrombopoiesis with many mononuclear cells resembling abnormal megakaryoblasts 

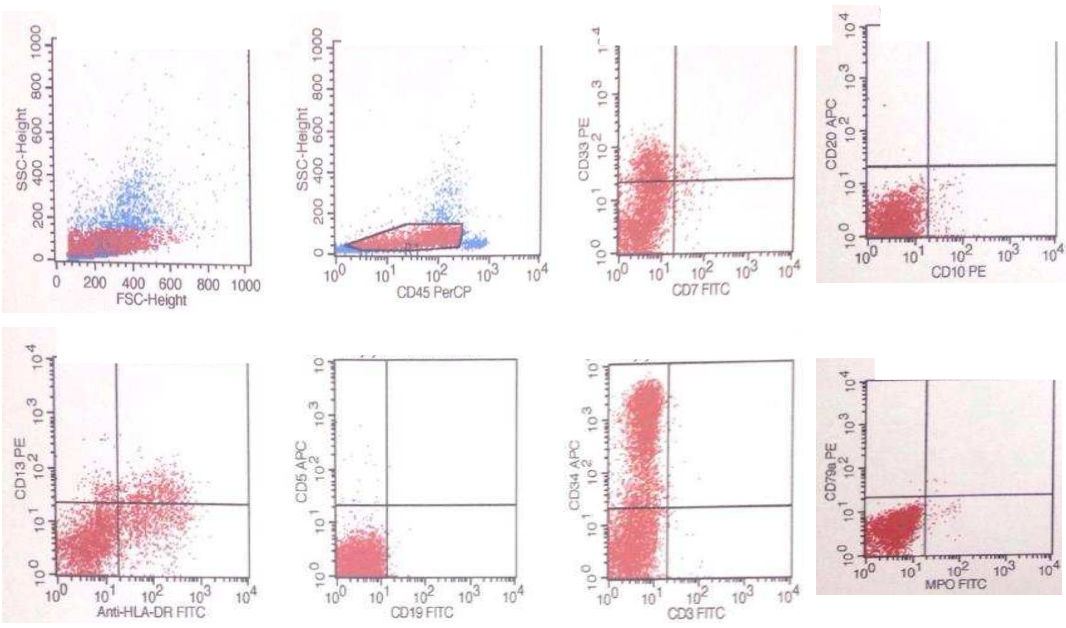

Figure 4. Immunophenotyping results on July 1, 2019 show CD33+, CD34+, CD13+ Conclusion: the gating blast area shows myeloid lineage
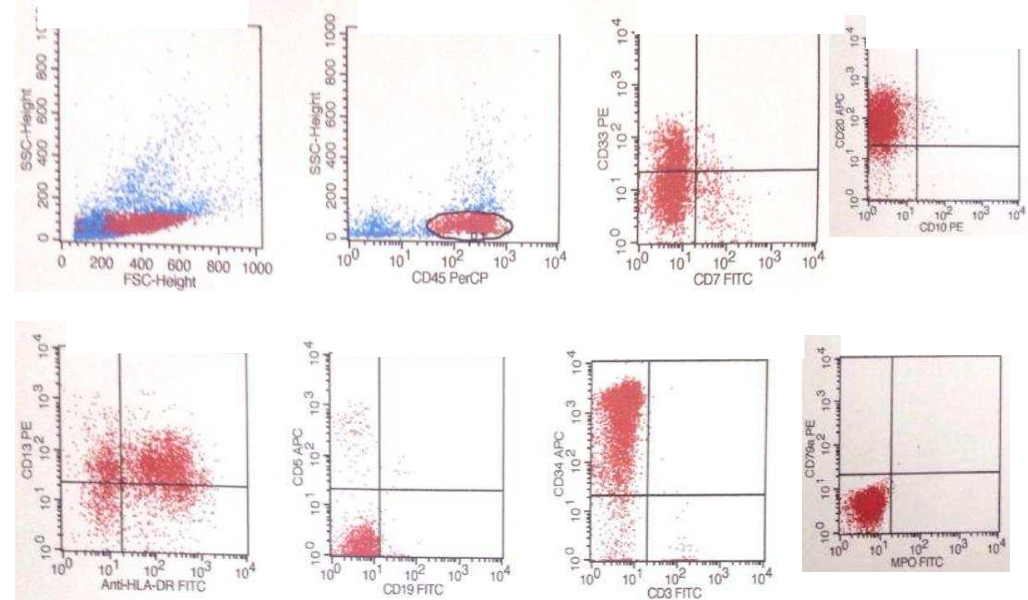

Figure 5. Immunophenotyping results on August 6, 2019 show CD33+, CD34+, CD13+, CD 20+, HLA-DR + Conclusion: the gating blast area expresses myeloid lineage with an aberrant CD20

The patient's condition after the immunophenotyping examination on August 6, 2019 worsened and died.

\section{Discussion}

Blast crisis-phase CML can be diagnosed when there is a blast of $\geq 20 \%$ of the peripheral leukocyte count or nucleated cells in the bone marrow, or when there is extramedullary blast proliferation. The course of the change in CML from the chronic phase to the accelerated/blast phase takes four years (2). Our patient had splenomegaly and routine blood results showed hemoglobin $8.1 \mathrm{~g} / \mathrm{dL}$, leukocyte count 33,440/ $\mathrm{LL}$, platelet count $111,000 / \mu \mathrm{L}$, and analyse of White Blood Cell Differential (WDF) channel channel and White Precursor 
Cell (WPC) channel on the Sysmex XN-1000 (figure 1) suggest a myeloid blast series and immature granulocytes. Low hemoglobin and platelets and high leukocytes and basophils plus flagging blasts, immature granulocytes present indicate a chronic blastic crisis phase of leukemia.

In this case, the Immature Platelet Fraction (IPF) has a high value of $29.2 \%$. Thrombocytopenia in leukemia patients is caused by bone marrow suppression, resulting in decreased platelet production and low IPF values. However, in this case, a high IPF value was found, so the suspicion of immature platelets could not be ruled out.

Megakaryoblasts morphologically have a high nucleus to cytoplasm ratio, cytoplasmic budding, or vacuole. The size of the blast cells is quite variable with a round nucleus, slightly irregular nucleus shape or indented nucleus with fine reticular chromatin and one to three nucleoli. The cytoplasm is basophilic and usually shows a bleb or pseudopod form (1). The morphology of the peripheral blood smear of our patient revealed mononuclear cells with bluish cytoplasm with cytoplasmic blebs, loose chromatin and indistinct nuclei suggesting an abnormal micromegakaryocyte with a proportion of $76 \%$, neutrophil segment $1 \%$, basophil $2 \%$, lymphocytes $18 \%$, monocytes $3 \%$. Bone marrow morphology of our patients found increased megakaryopoiesis activity, megakaryocytes were easily found with dysplasia (+), many mononuclear cells were found that resembled abnormal megakaryoblasts with a proportion of $45 \%$. The morphological findings of our patient's peripheral blood and bone marrow smears were identical in both groups.

The basic characteristics of leukemogenesis according to Gilliland and Griffin require two driving mutations: the first is a class-II mutation as inhibition of differentiation and apoptosis, the second is a class-I mutation that provides a proliferative advantage. The BCR-ABL gene fusion in the pathogenesis of AML most likely represents a class-I mutation (3). Another test found in our patient was a positive p210 BCR ABL gene fusion. Differentiating acute megakaryoblastic leukemia (AMeL) from crisis blastic CML is of great importance as it has important implications in the management of these patients.

Confirmation of megakaryocyte-platelet lineage was confirmed by showing specific markers of megakaryocyte-platelet lineage, expression of CD41, CD42b, CD61, CD62, and Factor VIII (2). On the first immunophenotyping results of our patient was found CD33+, CD34+(37\%), CD13+(17\%) and on the second immunophenotyping was found CD33+, CD34+(66.54\%), CD13+, CD20+, HLA-DR+. In this case, a negative result for the lymphoid marker CD3, CD79a could rule out the presence of lymphoblasts. The suspicion of AML was confirmed by positive immunophenotyping of the myeloid lineage markers. In this case no specific marker of megakaryocyte-platelet lineage was examined due to the limitations of the flow cytometric immunophenotyping marker panel at our hospital, but the suspicion of CML transformation from blastic crisis to megakaryoblastic phase could not be ruled out.

Prognosis is significantly poor in blastic crisis phase CML patients with megakaryoblastic transformation (1). The use of Imatinib helps to return from the blastic crisis phase to the chronic phase, which may improve outcomes following stem cell transplantation (3).

\section{Conclusion}

Clinical features, morphology and cytogenic findings assist in the identification and differentiation of blastic crisis phase CML from AMegL. A patient with CML has been reported with suspicion of megakaryoblasts on the basis of bone marrow morphology examination and an elevated IPF.

\section{Acknowledgements}

The author would like to thank Mr. Paulus Budiono Notopuro, MD., PhD., who has helped and guided the writing of this case report. 


\section{References}

1. Khemka R, Gupta M, Jena NK. CML with Megakaryocytic Blast Crisis: Report of 3 Cases. Pathol Oncol Res. $2019 ; 25^{3}: 1253-8$.

2. Amriah Buang. WHO Klassifikation 2016. Blood [Internet]. 2006;2(20):58-71. Available from: http://www.ukm.my/geografia.

3. Neuendorff NR, Burmeister T, Dörken B, Westermann J. BCR-ABL-positive acute myeloid leukemia : a new entity? Analysis of clinical and molecular features. Ann Hematol [Internet]. 2016;1211-21. Available from: http://dx.doi.org/10.1007/s00277-0162721-z.

4. Karkuzhali P, Shanthi V, Usha T. A case of chronic myeloid leukaemia presenting as megakaryocytic blast crisis (AML M7). Ecancermedicalscience. 2013;7:375:3-8. 